\title{
Atomic-scale control of competing electronic phases in ultrathin $\mathrm{LaNiO}_{3}$
}

\author{
P. D. C. King, ${ }^{1,2}$ H. I. Wei, ${ }^{2}$ Y. F. Nie, ${ }^{2,3}$ M. Uchida,${ }^{2}$ C. Adamo, ${ }^{3}$ \\ S. Zhu ${ }^{3}$ X. He, ${ }^{4}$ I. Božović, ${ }^{4}$ D. G. Schlom,,${ }^{1,3}$ and K. M. Shen ${ }^{1,2, *}$ \\ ${ }^{1}$ Kavli Institute at Cornell for Nanoscale Science, Ithaca, New York 14853, USA \\ ${ }^{2}$ Laboratory of Atomic and Solid State Physics, Department of Physics, \\ Cornell University, Ithaca, New York 14853, USA \\ ${ }^{3}$ Department of Materials Science and Engineering, \\ Cornell University, Ithaca, New York 14853, USA \\ ${ }^{4}$ Brookhaven National Laboratory, Upton, New York 11973-5000, USA
}

(Dated: February 17, 2014) 
In the effort to scale down electronic devices to atomic dimensions,${ }^{1}$ the use of transition-metal oxides may provide advantages over conventional semiconductors. Their high carrier densities and short electronic length scales are desirable for miniaturisation, ${ }^{2}$ while strong interactions which mediate exotic phase diagrams $^{3}$ open new avenues for engineering emergent properties. ${ }^{4,5}$ Understanding how their correlated electronic states can be manipulated at the nanoscale, however, remains challenging. Here, we use angle-resolved photoemission spectroscopy to uncover an abrupt destruction of Fermi liquid-like quasiparticles in the correlated metal $\mathrm{LaNiO}_{3}$ when confined to a critical film thickness of 2 unit cells. This is accompanied by the onset of an insulating phase as measured by electrical transport. We show how this is driven by an instability to an incipient order of the underlying quantum many-body system, demonstrating the power of artificial confinement to harness control over competing phases in complex oxides with atomic-scale precision.

Correlated transition-metal oxides host a diverse array of electronic and magnetic properties, including high-temperature superconductivity, quantum magnetism, ferroelectricity, and Mott metal-insulator transitions (MITs). Their extreme sensitivity to dimensionality opens new routes to manipulate their collective ground states by confining their thickness to the nanometer scale. Unlike other atomically thin materials such as graphene, however, their properties typically cannot be accurately predicted by ab-initio density-functional theory (DFT) due to strong electron-electron correlations. A major challenge of theoretical condensed matter physics is to accurately treat these interactions. Approximations such as dynamical mean-field theory are still extremely expensive and difficult for nanoscale systems, motivating experimental approaches which combine tools to engineer complex oxides with atomic-scale precision with probes to simultaneously reveal their electronic structure and the many-body interactions at the heart of their subtle quantum phases.

Here, we employ reactive-oxide molecular-beam epitaxy (MBE) to synthesise atomicallydefined layers of the correlated metal $\mathrm{LaNiO}_{3}$ down to just one pseudocubic unit cell (u.c.) thickness, epitaxially stabilised on $\mathrm{LaAlO}_{3}(001)$ substrates. Combining in situ angle-resolved photoemission spectroscopy (ARPES) with electrical transport measurements, we uncover an instability towards an insulating ordered phase that abruptly onsets at 2 u.c. We use ARPES to directly image how the momentum-resolved electronic structure evolves through 
the thickness-driven MIT, shedding new light on how phase competition can be manipulated at the nanoscale by building up correlated quantum materials one atomic layer at a time.

Figure 1 summarises the bulk electronic structure of $\mathrm{LaNiO}_{3}$. The predominantly $\mathrm{Ni} e_{g^{-}}$ derived Fermi surface predicted by DFT (Fig. 1(a)) comprises large hole pockets centred at the Brillouin zone corners and a small electron pocket at the zone centre, in good agreement with ARPES from our thicker films and previous soft x-ray photoemission. ${ }^{6}$ From x-ray absorption $^{7}$ we expect no substantial orbital polarisation to be induced by the epitaxial strain of $\sim-1 \%$ imposed by our $\mathrm{LaAlO}_{3}$ substrates, consistent with our ARPES Fermi surfaces. While DFT predicts the Fermi surface well (Fig. 1(a) and Supplemental Fig. 9), our measured energy $(E)$ vs. momentum $(\mathbf{k})$ dispersions are strongly renormalised with respect to the calculations: the experimental bandwidth is a factor of 7 smaller than the calculated bare band, and the dispersions we extract from fits to momentum distribution curves near the Fermi level, $E_{\mathrm{F}}$, have a velocity 7 times smaller than in DFT (Fig. 1(b)). This indicates a pronounced effect of electron-electron interactions narrowing the occupied bandwidth and sufficiently enhancing the effective mass to place $\mathrm{LaNiO}_{3}$ on the borderline of a Mott transition. ${ }^{8}$

This is in contrast to previous soft x-ray ARPES measurements ${ }^{6}$ which reported negligible renormalisation of the hole-like bands, likely due to the modest energy resolutions that can be achieved using soft x-rays which, in Ref. ${ }^{6}$, exceeded our measured bandwidth by approximately a factor of three. Our measurements instead reveal an unusually strong mass enhancement of these carriers, consistent with heavy quasiparticle masses determined from thermodynamic and optical studies ${ }^{9-13}$ and in qualitative agreement with recent dynamical mean-field theory calculations. ${ }^{14}$ The electronic structure nonetheless remains characteristic of a model Fermi liquid (Fig. 1(d)), with a frequency $(\omega)$ dependence of the scattering rate that goes as $\omega^{2}$ (Fig. 1(c)) and no large dispersion anomalies or vertical tails of incoherent spectral weight as often observed in other correlated materials. ${ }^{15}$

We track how this strongly-interacting electronic structure evolves as we confine $\mathrm{LaNiO}_{3}$ to ultrathin dimensions where both quantum size effects and electron correlations would be expected to become progressively more dominant. While previous studies have found a gradual crossover to an insulating state by 5 u.c. thickness or above, ${ }^{16-19}$ we find that the bulk-like electronic structure and Fermi liquid characteristics remain almost unaffected by film thickness down to 3 u.c., consistent with persistent metallicity which we observe in 
transport (Fig. 2). At 3 u.c., this is the thinnest metallic nickelate reported to date. ${ }^{16-19}$ This reflects the high sample quality, supported by high residual resistivity ratios $(R R R=$ $\rho(300 K) / \rho(4 K)$, where $\rho(T)$ is the measured resistivity at temperature $T)$ which exceed 11 at 25 u.c. thickness. This is similar to the best values achieved in bulk $\mathrm{LaNiO}_{3},{ }^{20}$ and higher than typically found for epitaxial nickelate films. It indicates low levels of disorder such as cation off-stoichiometry or bulk oxygen vacancies, which we confirm using postgrowth high-pressure oxygen annealing (see Supplementary Discussion 1), enabling metallic transport even in ultrathin 3 u.c. films. Reducing the thickness by just one further u.c., however, causes the ARPES spectral weight near $E_{\mathrm{F}}$ to be suddenly suppressed, the occupied bandwidth to be substantially broadened, and the films to become insulating by transport. For a single $\mathrm{LaNiO}_{3}$ layer, the resistivity becomes too high to be measured with our apparatus and we observe no spectral weight at the Fermi level, indicating the opening of a full charge gap. While the resistivity of 2 u.c. films decrease following high-pressure oxygen annealing, healing some surface oxygen vacancies and time-dependent film degradation, they remain insulating. This confirms that oxygen vacancies do not drive the MIT observed here ${ }^{21}$ (see Supplementary Discussion 1). Moreover, the direct correlation between the transport and spectroscopic properties of our films strongly supports that we have uncovered an intrinsic thickness-driven transition in 2 u.c. thick $\mathrm{LaNiO}_{3}$ epitaxial films, consistent with a MIT observed in bilayer $\mathrm{LaNiO}_{3} / \mathrm{LaAlO}_{3}$ superlattices. $^{5}$

Our momentum-resolved spectroscopic observations allow us to rule out several further possible causes of this MIT. The bandwidth is independent of film thickness from 50 down to 3 u.c. (Fig. 3(b)), incompatible with a Mott transition in the Brinkman-Rice scenario where a smooth divergence of the effective mass would be expected. This also rules out the possibility of bandwidth narrowing by quantum size effects, which would again be expected to induce more gradual changes in the electronic structure. ${ }^{22,23}$ We can exclude that strong localisation $^{16}$ is responsible for the MIT in our samples. Our resistivity measurements do show signs of weak localisation at 3 u.c. below $35 \mathrm{~K}$, but the inelastic mean free path of carriers that we estimate from momentum distribution curve peak widths at the Fermi level is largely thickness independent, remaining above $8 \AA$ even for the 2 u.c. sample, comparable with the film thickness and easily exceeding the Ioffe-Regel $\operatorname{limit}^{24}$ expected for a localisation-driven MIT.

Rather, our measurements reveal that the MIT is correlated with a sudden breakdown of 
Fermi liquid-like quasiparticles at 2 u.c. As evidenced by energy distribution curves at the Fermi wavevector, $k_{\mathrm{F}}$ (Fig. $3(\mathrm{a})$ ), samples $\geq 3$ u.c. support coherent quasiparticle-like excitations, indicated by the pronounced peak at $E_{\mathrm{F}}$, with only a small incoherent tail at higher binding energies. In contrast, decreasing the film thickness to 2 u.c. causes a pronounced suppression of the coherent peak, with almost all of the spectral weight transferred to incoherent excitations at higher binding energies. To describe this quantitatively, we define an effective quasiparticle residue, $Z^{\prime}$ (Fig. 3(b), see Supplemental Discussion 2). We find this to be independent of film thickness down to 3 u.c., below which it suddenly drops nearly to zero. We note that a temperature-dependent loss of quasiparticle coherence has previously been associated with an onset of insulating $c$-axis transport in several layered oxides. ${ }^{25}$ Our measurements here show that a similar mechanism can lead to a dimensionality-driven MIT in ultrathin correlated metals.

These findings are in striking contrast to previous photoemission studies which suggested a much more gradual loss of quasiparticle weight starting at films $\geq 10$ u.c. ${ }^{18,19}$ with its total suppression by $\sim 4$ u.c. While such a slow crossover was attributed to gradual localisation, ${ }^{19}$ our sharp momentum distribution curves at $E_{\mathrm{F}}$ as well as the sudden collapse of quasiparticle residue we find at 2 u.c. instead points to an instability of $\mathrm{LaNiO}_{3}$ to an underlying charge/spin order in two dimensions. The total loss of weight of the $e_{g}$ states is accompanied by a shift of the occupied $t_{2 g}$ and $\mathrm{O} 2 p$ states to higher binding energies in our single u.c. sample (Fig. 2(g)), notably similar to that observed through the temperature-driven MIT into a charge-ordered ground state in $\mathrm{NdNiO}_{3} \cdot{ }^{27}$ This suggests that a similar charge and/or spin ordering triggers the thickness-driven MIT we observe in $\mathrm{LaNiO}_{3}$.

We note that experimental signatures of spin order, accompanying a MIT, have recently been observed in dimensionally-confined bilayer $\mathrm{LaNiO}_{3} / \mathrm{LaAlO}_{3}$ superlattices, ${ }^{5,26}$ while bulk $R E_{x} \mathrm{Sr}_{2-x} \mathrm{NiO}_{4}$ with $x \sim 1$ (a quasi-2D analogue of $\mathrm{LaNiO}_{3}$ ) lies on the borderline of metallic and insulating charge- and spin-ordered phases. ${ }^{28}$ Unlike mesoscopic superlattices or macroscopic bulk crystals, however, our work demonstrates how control between such ordered insulating and metallic phases can be achieved in a material of total thickness on the order of only $1 \mathrm{~nm}$. This highlights the power of dimensional engineering in ultrathin correlated oxides and suggests its potential for controlling novel electronic phases for use in functional nanoscale electronics. Recent calculations further support this picture, suggesting a pronounced stabilisation of magnetically-ordered insulating phases in bilayer (2 u.c.) and 
monolayer (1 u.c.) $\mathrm{LaNiO}_{3}$ as compared to film thicknesses $\geq 3$ u.c. ${ }^{29}$ in excellent agreement with the critical thickness for the MIT observed here.

Whether these ordering tendencies could be enhanced by structural modification in ultrathin films remains an open question. Increased octahedral tilts have been reported in the bottom layer of $\mathrm{LaNiO}_{3}$-based superlattices, ${ }^{30}$ polar distortions may cause small structural rumplings close to the $\mathrm{LaNiO}_{3}$ surface, ${ }^{31}$ and out-of-plane Ni-O bond lengths could be modified at the $\mathrm{LaNiO}_{3}$ /vacuum interface. An insulating surface resulting from such reconstruction or bond length disproportionation is not easily reconciled with our surface-sensitive measurements of the thicker films, and we find no evidence for increased octahedral rotations in our thinnest films from electron diffraction (Supplementary Fig. 7). Nonetheless, we do not rule out the presence of small structural distortions which could drive the system closer to the ordered phases characteristic of the sister rare-earth nickelates.. ${ }^{32}$

Even in the bulk, however, our measured Fermi surfaces exhibit strong in-plane nesting with wavevectors $|\mathbf{q}| \approx 0.3 \pi / a$ where $a$ is the in-plane lattice constant, consistent with a stripe- or checkerboard-type ordering instability of the underlying electronic system. ${ }^{33}$ Our ARPES measurements reveal a downward shift of the band bottom at 2 u.c. (Fig. 3(b)), which would not be expected for a Mott transition or localisation, but is reminiscent of the formation of a density wave, where corresponding gapping of the Fermi surface could drive a MIT. We stress, however, that the simplest weak coupling scenarios are insufficient to fully explain our data. These would not give rise to the abrupt loss in quasiparticle coherence we find at 2 u.c., while we observe no back-bending of the bands near $E_{\mathrm{F}}$, a weak spectral signature of density-wave order, but one that is typically not observed in the strong-coupling limit even for systems with robust static order. Rather, our measurements for 2 u.c. film thickness, showing an incoherent band with residual spectral weight up to the Fermi level and rather low, albeit still insulating, absolute values of the resistivity, point to the presence of strongly fluctuating order in the bilayer (Fig. 4(b)), and its close proximity to the MIT. We thus expect short correlation lengths and a concomitant loss of quasiparticle coherence. In contrast, the lack of any spectral weight at the Fermi level and high resistivity of the single unit cell sample suggests freezing of this order in the true two-dimensional limit (Fig. 4(b)).

Our measurements therefore reveal how the phase diagram of $\mathrm{LaNiO}_{3}$ can be manipulated by controlling the number of electronically-active $\mathrm{NiO}_{2}$ planes (Fig. 4(a)). We have shown how a resilient bulk-like correlated metal phase gives way to fluctuating and then static 
ordered insulators for double and single layers, respectively. A 2 u.c. sample is the first for which no Ni atom exists within a bulk-like environment for nearest-neighbour hopping along the surface normal direction (Fig. 4(b)), ultimately driving its susceptibility to the incipient order of the two-dimensional limit. Finite inter-layer hopping, however, allows fluctuations to play an essential role, placing this quasi two-dimensional compound right at the border between paramagnetic metallic (3 u.c.) and ordered insulating (1 u.c.) phases. Our work thus demonstrates the power of precise layer-by-layer control for tuning the delicate balance between competing phases in correlated electron materials, opening the door towards an exotic new generation of atomic-scale oxide electronics. 


\section{Methods}

Reactive-oxide molecular-beam epitaxy: Epitaxial films of $\mathrm{LaNiO}_{3}$ were grown on (001) pseudocubic $\mathrm{LaAlO}_{3}$ substrates by reactive-oxide molecular-beam epitaxy (MBE) in a Veeco GEN10 system equipped with reflection high-energy electron diffraction (RHEED), and utilising a high background partial pressure of $10^{-5}$ Torr of distilled ozone as an oxidant during growth. Our La and Ni effusion cells were mounted in differentially-pumped enclosures (Veeco retractable differentially-pumped sources) to prevent source oxidation during growth, and maintain good flux stability. Samples were grown at a temperature of $\sim 550^{\circ} \mathrm{C}$, using alternating monolayer depositions of $\mathrm{LaO}$ and $\mathrm{NiO}_{2}$, followed by a $20 \mathrm{~s}$ annealing time. Further details of our growth procedure and sample characterisation are presented in the Supplementary Discussion 1.

Angle-resolved photoemission spectroscopy: After growth, the samples were cooled in ozone to $250^{\circ} \mathrm{C}$, and then immediately transferred through ultrahigh vacuum to our ARPES chamber with a base pressure better than $5 \times 10^{-11}$ Torr. The total transfer duration was $\sim 300$ s. ARPES measurements were then performed using a VG Scienta R4000 hemispherical electron analyser and a monochromatised VUV5000 helium plasma lamp. One of the Fermi surface maps in Fig. 1(a) was measured using the $\mathrm{He} \mathrm{II} \alpha$ line $(h \nu=40.8 \mathrm{eV}$ where $h$ is Planck's constant and $\nu$ the light frequency). All other measurements shown here were performed using He $\mathrm{I} \alpha$ radiation $(h \nu=21.2 \mathrm{eV})$. The measurement temperature was $20 \mathrm{~K}$ for the data shown from film thicknesses of 3, 5, 8, 25, and 50 u.c., $50 \mathrm{~K}$ for 2 and 4 u.c., and $330 \mathrm{~K}$ for the single u.c. thick sample to prevent charging. In all cases, we experimentally confirmed that the samples were not charging by performing beam flux- and temperature-dependent measurements. 
* E-mail: kmshen@cornell.edu.

[1] Britnell, L. et al. Field-Effect Tunneling Transistor Based on Vertical Graphene Heterostructures. Science 335, 947-950 (2012).

[2] Takagi, H. \& Hwang, H. Y. An emergent change of phase for electronics. Science 327, 1601-1602 (2010).

[3] Dagotto, E. Complexity in Strongly Correlated Electronic Systems. Science 309, 257-262 (2005).

[4] Hwang, H. Y. et al. Emergent phenomena at oxide interfaces. Nature Mater. 11, 103-113 (2012).

[5] Boris, A. V. et al. Dimensionality Control of Electronic Phase Transitions in Nickel-Oxide Superlattices. Science 332, 937-940 (2011).

[6] Eguchi, R. et al. Fermi surfaces, electron-hole asymmetry, and correlation kink in a threedimensional Fermi liquid $\mathrm{LaNiO}_{3}$. Phys. Rev. B 79, 115122 (2009).

[7] Chakhalian, J. et al. Asymmetric Orbital-Lattice Interactions in Ultrathin Correlated Oxide Films. Phys. Rev. Lett. 107, 116805 (2011).

[8] Qazilbash, M. M. et al. Electronic correlations in the iron pnictides. Nature Phys. 5, 647-650 (2009).

[9] Rajeev, K., Shivashankar, G. \& Raychaudhuri, A. Low-temperature electronic properties of a normal conducting perovskite oxide $\mathrm{LaNiO}_{3}$. Solid. State. Commun. 79, 591-595 (1991).

[10] Xu, X. Q., Peng, J. L., Li, Z. Y., Ju, H. L. \& Greene, R. L. Resisitivity, thermopower, and susceptibility of $R \mathrm{NiO}_{3}$ (R=La,Pr). Phys. Rev. B 48, 1112-1118 (1993).

[11] Sreedhar, K. et al. Electronic properties of the metallic perovskite $\mathrm{LaNiO}_{3}$ : Correlated behavior of $3 d$ electrons. Phys. Rev. B 46, 6382-6386 (1992).

[12] Ouellette, D. G. et al. Optical conductivity of $\mathrm{LaNiO}_{3}$ : Coherent transport and correlation driven mass enhancement. Phys. Rev. B 82, 165112 (2010).

[13] Stewart, M. K. et al. Optical study of strained ultrathin films of strongly correlated $\mathrm{LaNiO}_{3}$. Phys. Rev. B 83, 075125 (2011).

[14] Deng, X. et al. Hallmark of strong electronic correlations in $\mathrm{LaNiO}_{3}$ : Photoemission kink and broadening of fully occupied bands. Phys. Rev. B 85, 125137 (2012). 
[15] Meevasana, W. et al. Hierarchy of multiple many-body interaction scales in high-temperature superconductors. Phys. Rev. B 75, 174506 (2007).

[16] Scherwitzl, R. et al. Metal-Insulator Transition in Ultrathin $\mathrm{LaNiO}_{3}$ Films. Phys. Rev. Lett. 106, 246403 (2011).

[17] Son, J. et al. Low-dimensional Mott material: Transport in ultrathin epitaxial $\mathrm{LaNiO}_{3}$ films. Appl. Phys. Lett. 96, 062114 (2010).

[18] Gray, A. X. et al. Insulating state of ultrathin epitaxial $\mathrm{LaNiO}_{3}$ thin films detected by hard x-ray photoemission. Phys. Rev. B 84, 075104 (2011).

[19] Sakai, E. et al. Gradual localization of $\mathrm{Ni} 3 d$ states in $\mathrm{LaNiO}_{3}$ ultrathin films induced by dimensional crossover. Phys. Rev. B 87, 075132 (2013).

[20] Lacorre, P. et al. Synthesis, crystal structure, and properties of metallic $\mathrm{PrNiO}_{3}$ : Comparison with metallic $\mathrm{NdNiO}_{3}$ and semiconducting $\mathrm{SmNiO}_{3}$. J. Solid State Chem. 91, 225-237 (1991).

[21] Horiba, K. et al. Electronic structure of $\mathrm{LaNiO}_{3}$ : An in situ soft x-ray photoemission and absorption study. Phys. Rev. B 76, 155104 (2007).

[22] Yoshimatsu, K. et al. Metallic Quantum Well States in Artificial Structures of Strongly Correlated Oxide. Science 333, 319-322 (2011).

[23] Meevasana, W. et al. Creation and control of a two-dimensional electron liquid at the bare $\mathrm{SrTiO}_{3}$ surface. Nature Mater. 10, 114-118 (2011).

[24] Ioffe, A. F. \& Regel, A. R. Non-crystalline, amorphous, and liquid electronic semiconductors. Prog. Semicond. 4, 237 (1960).

[25] Valla, T. et al. Coherence-incoherence and dimensional crossover in layered strongly correlated metals. Nature 417, 627-630 (2002).

[26] Frano, A. et al. Orbital Control of Noncollinear Magnetic Order in Nickel Oxide Heterostructures. Phys. Rev. Lett. 111, 106804 (2013).

[27] Schwier, E. F. et al. Unusual temperature dependence of the spectral weight near the Fermi level of $\mathrm{NdNiO}_{3}$ thin films. Phys. Rev. B 86, 195147 (2012).

[28] Uchida, M. et al. Pseudogap of Metallic Layered Nickelate $\mathrm{R}_{2-x} \mathrm{Sr}_{x} \mathrm{NiO}_{4}(R=\mathrm{Nd}$,Eu) Crystals Measured Using Angle-Resolved Photoemission Spectroscopy. Phys. Rev. Lett. 106, 027001 (2011).

[29] Lau, B. \& Millis, A. J. Theory of the magnetic and metal-insulator transitions in $r$ nio 3 bulk and layered structures. Phys. Rev. Lett. 110, 126404 (2013). 
[30] Hwang, J. et al. Structural origins of the properties of rare earth nickelate superlattices. Phys. Rev. B 87, 060101 (2013).

[31] Chen, H. et al. Modifying the electronic orbitals of nickelate heterostructures via structural distortions. Phys. Rev. Lett. 110, 186402 (2013).

[32] Torrance, J. B., Lacorre, P., Nazzal, A. I., Ansaldo, E. J. \& Niedermayer, C. Systematic study of insulator-metal transitions in perovskites $\mathrm{RNiO}_{3}(\mathrm{R}=\mathrm{Pr}, \mathrm{Nd}, \mathrm{Sm}, \mathrm{Eu})$ due to closing of charge-transfer gap. Phys. Rev. B 45, 8209-8212 (1992).

[33] Evtushinsky, D. V. et al. Bridging Charge-Orbital Ordering and Fermi Surface Instabilities in Half-Doped Single-Layered Manganite $\mathrm{La}_{0.5} \mathrm{Sr}_{1.5} \mathrm{MnO}_{4}$. Phys. Rev. Lett. 105, 147201 (2010).

Acknowledgements: This work was supported by the Office of Naval Research (Grant No. N00014-12-1-0791), the National Science Foundation through the MRSEC program (Cornell Center for Materials Research, DMR-1120296), and was performed in part at the Cornell NanoScale Facility, a member of the National Nanotechnology Infrastructure Network, which is supported by the National Science Foundation (Grant ECCS-0335765). X. He and I. Božović were supported by the U.S. Department of Energy, Basic Energy Sciences, Materials Sciences and Engineering Division. H.I. Wei acknowledges support from the NSF IGERT program (DGE-0654193). We are grateful to A. Georges, C.A. Marianetti, A.J. Millis, J.A. Mundy, and T.W. Noh for enlightening discussions.

Author contributions: The samples were grown by P.D.C.K. and C.A., and characterised by P.D.C.K., H.I.W., and Y.F.N. ARPES measurements were performed by P.D.C.K, H.I.W, Y.F.N, and M.U, and analysed by P.D.C.K. S.Z grew the $\mathrm{LaAlO}_{3}$ buffer layers used for some samples. X.H. and I.B. performed the high-pressure oxygen annealing experiments and corresponding sample characterisation. P.D.C.K and K.M.S. wrote the paper, with input and discussion from all co-authors. P.D.C.K, D.G.S., and K.M.S. devised the project, and were responsible for its overall planning and direction.

Additional Information: The authors declare no competing financial interests. Supplementary information accompanies this paper at www.nature.com/naturenanotechnology. Reprints and permission information is available online at http://npg.nature.com/reprintsandpermissions/. Correspondence and requests for materials should be addressed to K.M.S. 
Figure captions:

FIG. 1. Giant quasiparticle mass renormalisation in $\mathbf{L a N i O}_{3}$. (a) Three-dimensional Fermi surface of bulk $\mathrm{LaNiO}_{3}$, predominantly derived from Ni $e_{g}$ orbitals, as predicted by densityfunctional (DFT) calculations (see Supplemental Discussion 3). Our experimental measurements probe almost two-dimensional projections about the planes shown through the bulk Brillouin zone. Corresponding simulations derived from our calculations (bottom left) are well matched by our experimental ARPES measurements (bottom right) of a 50 pseudocubic unit cell (u.c.) sample measured using $\mathrm{He} \mathrm{I} \alpha\left(k_{z} \approx 0.7 \pi / a\right)$ and $\mathrm{He} \mathrm{II} \alpha\left(k_{z} \approx 0.3 \pi / a\right)$ light (see also Supplementary Fig. 9). (b) The measured $E$ vs. $\mathbf{k}$ dispersions, shown for an 8 u.c. sample along the red cut in (a), are renormalised by a factor of 7 from the corresponding DFT calculations, as illustrated by comparing fits to momentum distribution curves with the scaled DFT calculation on the magnified view shown inset. (c) The imaginary part of the self-energy extracted from momentum distribution curve peak widths and the (background subtracted) energy distribution curve at $k_{\mathrm{F}}$ are both well described by the same analytic self-energy of a three-dimensional Fermi liquid (green lines, $\left.\operatorname{Im}[\Sigma(\omega)]=\beta\left(\omega^{2}+\left(\pi k_{B} T\right)^{2}\right)+\Sigma_{\mathrm{imp}}, \beta=79 \mathrm{eV}^{-1}, \Sigma_{\mathrm{imp}}=0.27 \mathrm{eV}\right)$. The error bars shown reflect the uncertainty in extracting the imaginary part of the self energy from our fits to momentum distribution curves, incorporating statistical errors in peak fitting, systematic errors and sample-to-sample variations. Together, our spectroscopic measurements are indicative of the spectral function, $A(\mathbf{k}, \omega)$, of a model strongly-correlated Fermi liquid, as shown in (d) by energy distribution curves (magnified near $E_{\mathrm{F}}$ in the inset) simulated from our bare DFT band assuming a Fermi liquid self energy with a mass renormalisation of 7 and a $\beta$ term of $79 \mathrm{eV}^{-1}$ as in our experimental measurements. The impurity term has been set to $20 \mathrm{meV}$ to aid visualisation. 
FIG. 2. Thickness-driven metal-insulator transition. (a) Transport measurements reveal a MIT at a critical film thickness of 2 u.c. The evolution of the electronic structure across this MIT is followed by angle-resolved ( $\mathrm{b}-\mathrm{f}$ ) and angle-integrated $(\mathrm{g})$ photoemission spectroscopy along $\left(0.5 \pi / a, k_{y}, 0.7 \pi / a\right)$, for samples varying from $\left[\mathrm{LaNiO}_{3}\right]_{8}$ u.c. $/ \mathrm{LaAlO}_{3}$ to $\left[\mathrm{LaNiO}_{3}\right]_{1}$ u.c. $/ \mathrm{LaAlO}_{3}$, represented schematically by the structural motifs shown to the left and right of (g) and (h), respectively [Blue: Ni, Green: La, Red: O, Orange: Al]. These reveal a sudden transition from a narrow parabolic band characteristic of a strongly-correlated Fermi liquid metal ( $\geq 3$ u.c.) to a broad dispersive band with a pronounced loss of quasiparticle coherence (2 u.c.) to a fully gapped insulator (1 u.c.). The angle-resolved spectra were background subtracted as described in Supplementary Discussion 2. The inset to $(\mathrm{g})$ shows the near- $E_{\mathrm{F}}$ angle-integrated spectra on a magnified scale, together with the corresponding thickness-dependent peak shifts (blue arrows) described in the main text. (h) X-ray diffraction and atomic force microscopy (shown inset for a 4 u.c. sample, see also Supplementary Fig. 6) indicate the high structural quality of the samples, and the absence of islanding down to the thinnest films.

FIG. 3. Loss of quasiparticle integrity across the MIT. (a) Energy distribution curves at $k_{\mathrm{F}}$ taken from the ARPES spectra in Fig. 2 reveal pronounced quasiparticle-like peaks at the Fermi level for film thicknesses of 3 u.c. and above, which are almost completely suppressed at a critical thickness of 2 u.c. (b) This sudden crossover is evident from the effective quasiparticle residue, $Z^{\prime}$ (see Supplemental Discussion 2) and is accompanied by a large increase in binding energy of the bottom of the occupied band, as extracted from the ARPES data in Fig. 2. The error bars for $Z^{\prime}$ and the band bottom in (b) reflect the uncertainty in extracting peak areas of the coherent and incoherent components, and of the band bottom position, of the $k_{\mathrm{F}}$ and $k=0$ energy distribution curves, respectively. They include statistical errors in peak fitting, systematic experimental uncertainties, and sample-to-sample variations. 
FIG. 4. Atomic-scale control of competing quantum phases in ultrathin $\mathrm{LaNiO}_{3}$. (a) Phase diagram of $\mathrm{LaNiO}_{3}$ as a function of film thickness, reconstructed from our spectroscopic and transport measurements. (b) Schematic representation of the consequent evolution of the electronic structure across this phase diagram. Plausible spin structures ${ }^{26}$ are shown as a function of film thickness, with fluctuations represented schematically by the light blue arrows. Note, however, that we do not distinguish between spin and charge order (or their combination) in our experimental measurements. Only the $\mathrm{Ni}$ atoms are shown; each pseudocubic unit cell contains one $\mathrm{NiO}_{2}$ layer. 


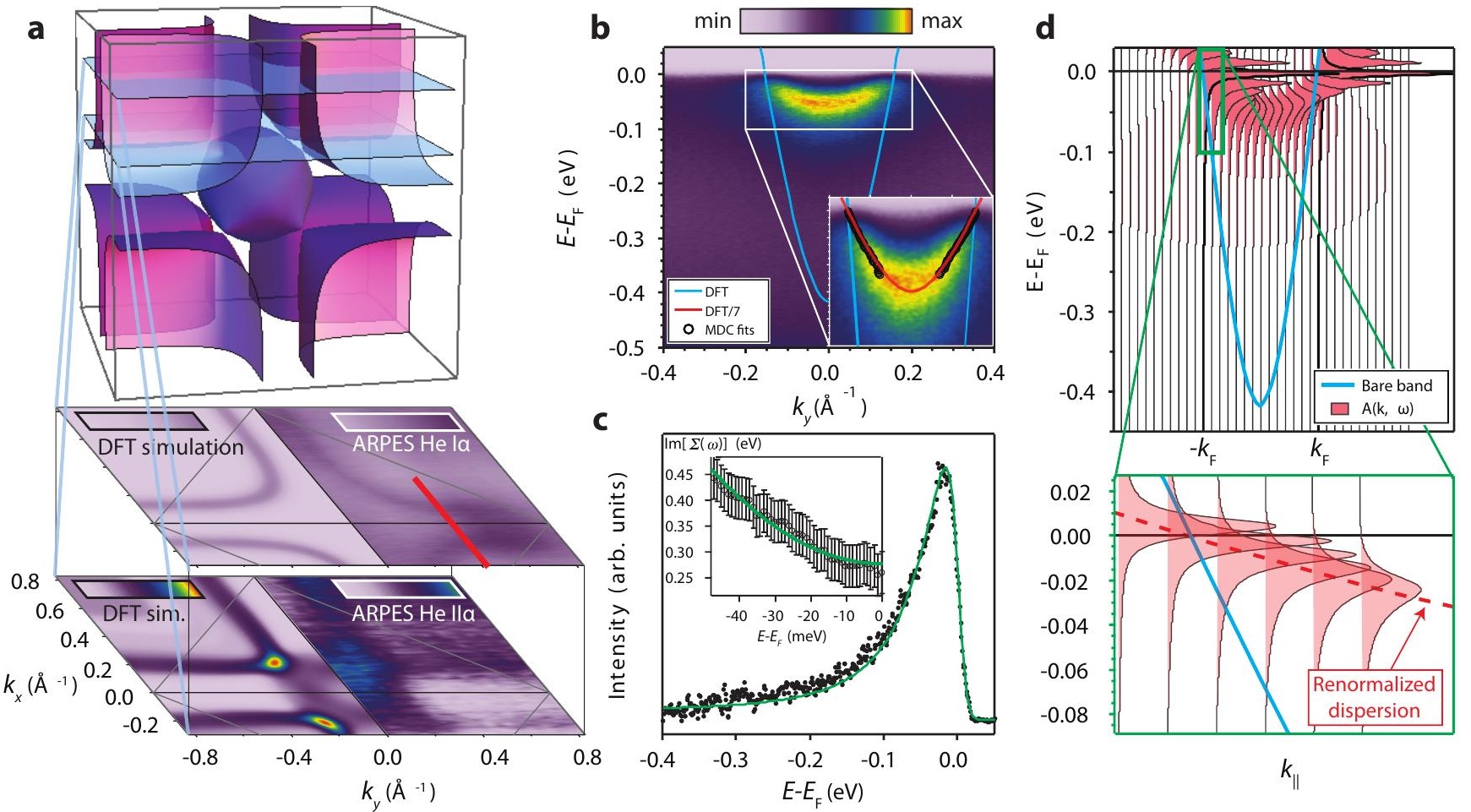




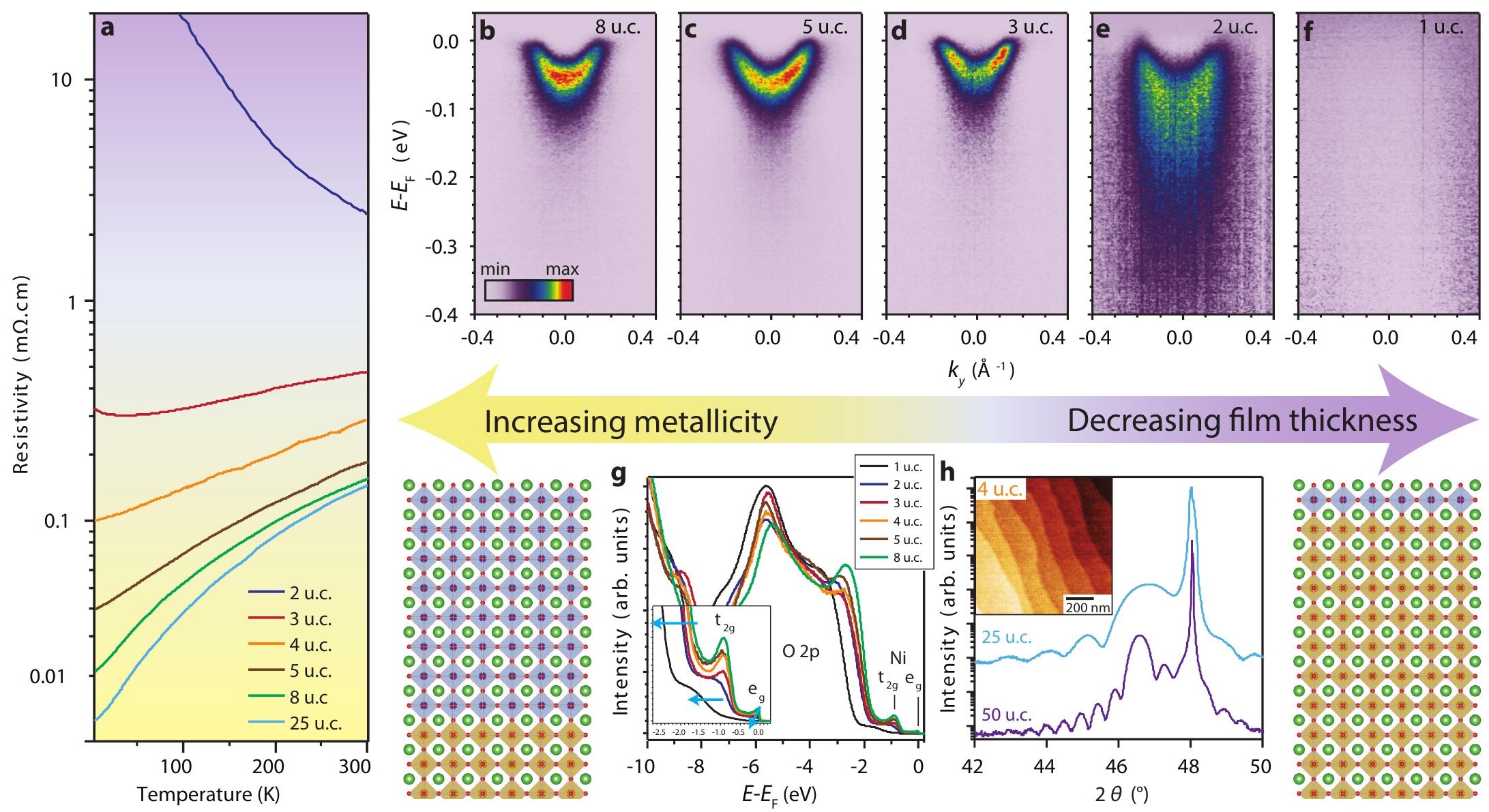


a

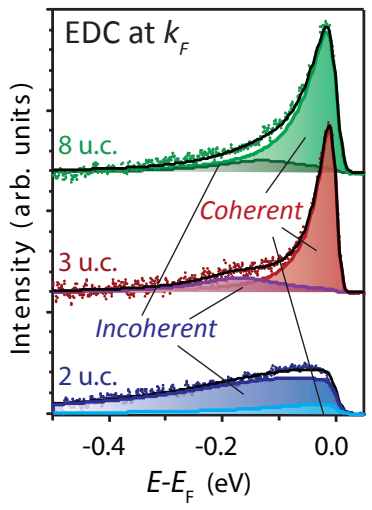

b

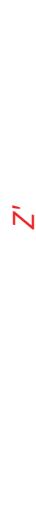

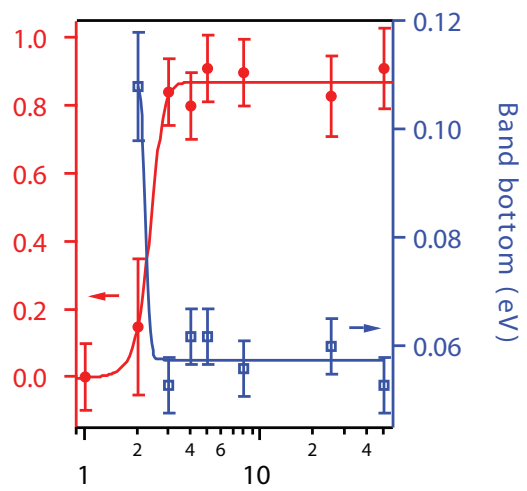

Film Thickness (u.c.) 
a

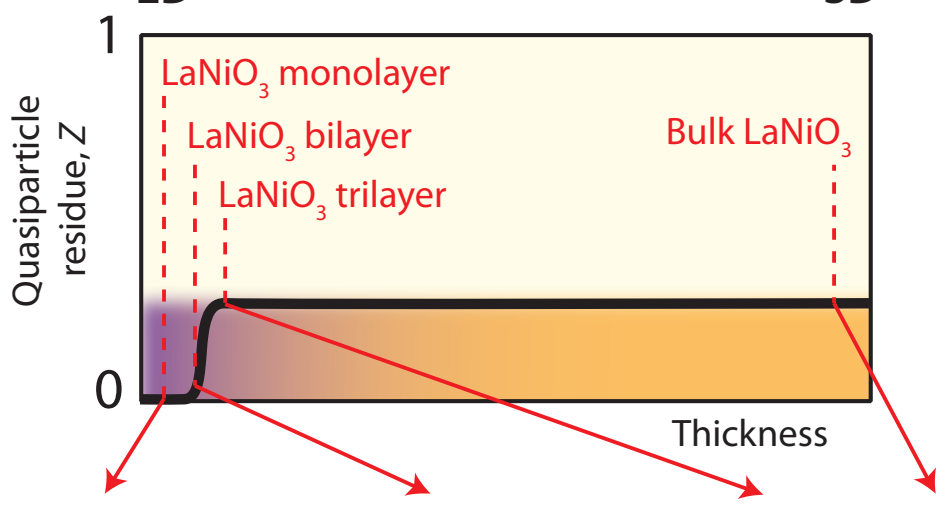

Static charge/spin order

Fully-gapped insulator
Incipient order Fluctuating Weak coupling
Strongly-interacting Fermi liquid metal

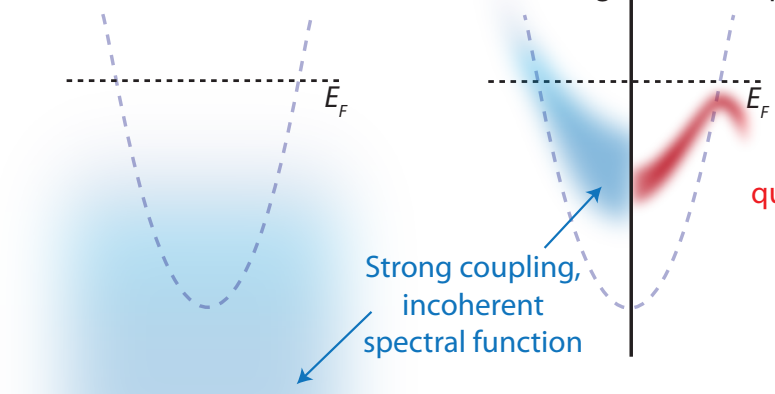

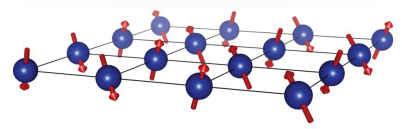

1 unit cell

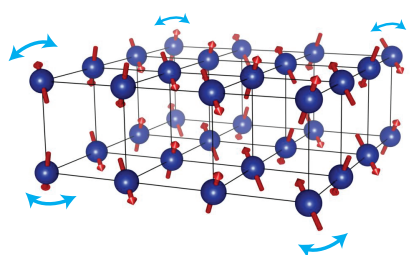

2 unit cells

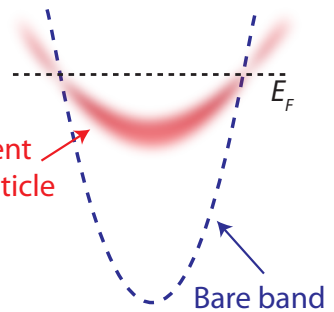
dispersion

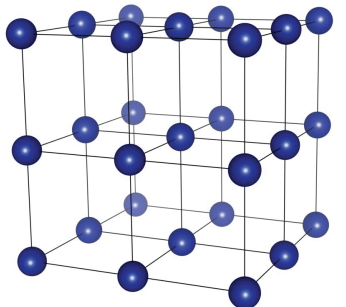

$\geq 3$ unit cells 


\title{
Supplementary Information: Atomic-scale control of competing electronic phases in ultrathin $\mathrm{LaNiO}_{3}$
}

\author{
P. D. C. King, H. I. Wei, Y. F. Nie, M. Uchida, C. Adamo, S. Zhu, X. He, I. Božović, D. G. Schlom, and K. M. Shen
}

(Dated: February 17, 2014)

\section{SUPPLEMENTARY DISCUSSION 1. SAMPLE GROWTH AND CHARACTERISATION}

Epitaxial films of $\mathrm{LaNiO}_{3}$ were grown on (001) pseudocubic $\mathrm{LaAlO}_{3}$ substrates by reactive-oxide molecularbeam epitaxy (MBE) in a Veeco GEN10 system equipped with reflection high-energy electron diffraction (RHEED), and utilizing distilled ozone as an oxidant. An $\mathrm{AlO}_{2}$-terminated surface with atomic step-and-terrace structure was achieved on the $\mathrm{LaAlO}_{3}$ substrates by a three-step procedure of boiling in DI water, ${ }^{1}$ annealing in oxygen, and re-boiling. For some samples, an additional homoepitaxial $\mathrm{LaAlO}_{3}$ buffer layer was grown before the $\mathrm{LaNiO}_{3}$ growth, but we found no systematic influence of this on either the structural or electronic properties of the $\mathrm{LaNiO}_{3}$ samples. In contrast, we found a very strong dependence on the ozone partial pressure during growth. Indeed, $\mathrm{LaNiO}_{3}$ is well known to be susceptible to oxygen vacancy formation. ${ }^{2}$ We therefore utilized high background partial pressures of $10^{-5}$ Torr of distilled ozone during growth. We additionally found relatively modest growth temperatures of $\sim 550^{\circ} \mathrm{C}$ were optimal, increasing the thermodynamic stability of the $\mathrm{Ni}^{3+}$ oxidation state.

To accurately determine the La-Ni stoichiometry, we grew a series of samples of approximately 15 pseudocubic unit cells (u.c.) thickness by co-depositing La and $\mathrm{Ni}$ in a background pressure of $10^{-5}$ Torr $\mathrm{O}_{3}$ and varied the relative flux of La: $\mathrm{Ni}$ by incrementing the $\mathrm{Ni}$ source temperature. Under near-stoichiometric conditions, we observed persistent RHEED oscillations (Fig. 1(a)), indicating a layer-by-layer growth mode is achieved. Moreover, we found that the residual resistivity ratio (RRR) of the resulting samples depended sensitively on the $\mathrm{La}-\mathrm{Ni}$ stoichiometry (Fig. 1(b)), serving as an excellent metric for the calibration of the relative flux of the two sources. Once flux-matched conditions are achieved, the period of RHEED oscillations directly gives the deposition time for a single unit cell of $\mathrm{LaNiO}_{3}$. To confirm this, we estimated the film thicknesses from Kiessig fringes observed in x-ray diffraction of our final thicker samples (see Fig. 2(h) of the main text). Using this estimate for the film thickness, a single period of oscillation of the RHEED intensity corresponds to $0.98 \pm 0.05$ unit cells. As a further check, we used the flux and monolayer deposition times determined from our $\mathrm{LaNiO}_{3}$ calibration to grow a sample of $\mathrm{La}_{2} \mathrm{NiO}_{4}$ by shuttered layer-by-layer deposition of $\left[\mathrm{LaO}-\mathrm{NiO}_{2}-\mathrm{LaO}\right]_{40}$. This is a sensitive test of both the relative and absolute monolayer dosage of La and $\mathrm{Ni}$. The high quality of the resulting x-ray diffraction from this sample (Fig. 1(c)) thereby confirms the accuracy of our calibration method for growth of stoi- chiometric $\mathrm{LaNiO}_{3}$.

We found that we could further improve the transport characteristics of our $\mathrm{LaNiO}_{3}$ films by utilizing an alternating shuttered, rather than co-incident, deposition of monolayers of $\mathrm{LaO}$ and $\mathrm{NiO}_{2}$, with an additional annealing time of $20 \mathrm{~s}$ following each $\mathrm{NiO}_{2}$ monolayer deposition (Fig. 2(a)). This results in an average growth rate of only $\sim 0.02 \AA / s$, which presumably further helps to limit the concentration of oxygen vacancies, optimizing the RRR of our samples. Previous bulk as well as $\sim 50$ u.c. thick epitaxial samples with RRRs of 3-4 were found to be fully oxygenated. ${ }^{3,4}$ Given the extreme sensitivity of $\mathrm{LaNiO}_{3}$ transport to oxygen vacancy creation, ${ }^{4}$ our RRR values, which exceed 11 at only 25 u.c. thickness (Fig. 3), suggests we have minimal concentrations of oxygen vacancies in the bulk of our samples.

To confirm this, we have performed extensive ex-situ annealing experiments of several 25 u.c. (metallic) and 2 u.c. (insulating) $\mathrm{LaNiO}_{3}$ samples, at high oxygen pressure up to $20 \mathrm{MPa}$ and at increasing temperatures up to $500^{\circ} \mathrm{C}$. Typical results are shown in Fig. 4, and were reproduced in other samples. For the 25 u.c. sample (Fig. 4(a)), we find almost no change in the transport properties for annealing up to $360^{\circ} \mathrm{C}$. This is followed by a very small decrease $(\sim 1 \%)$ in the room temperature resistivity after annealing at $375^{\circ} \mathrm{C}$, with no further changes observed for additional annealing cycles at higher temperatures. Qualitatively, we find similar results for the 2 u.c. sample (Fig. 4(b)), although with significantly larger relative changes. The resistivity of the as-grown film can be well fit by a simple exponential decay with a characteristic energy of $\sim 40 \mathrm{meV}$, consistent with density-wave formation resulting in a small semiconductor-like energy gap. This gap, and the roomtemperature resistivity, drops by up to $\sim 1$ order of magnitude following oxygen annealing, with the smallest resistivity found after annealing at $375^{\circ} \mathrm{C}$. Together, these results indicate that the structure becomes open to oxygen incorporation at temperatures around $375^{\circ} \mathrm{C}$ (at $p=20 \mathrm{MPa}$ of oxygen), a process which should heal oxygen vacancies. While this yields a 2 u.c. thick film close to a MIT, entirely consistent with our ARPES measurements shown in the main text, it remains unambiguously insulating indicating that the MIT at 2 u.c. is intrinsic and not driven by oxygen vacancies.

Indeed, the significantly higher relative changes observed in the 2 u.c. sample point to very low concentrations of oxygen vacancies in the bulk, while strongly suggesting that the samples possess a surface oxygendeficient region during the transport measurements. This cannot be present immediately after the growth, however, 
a
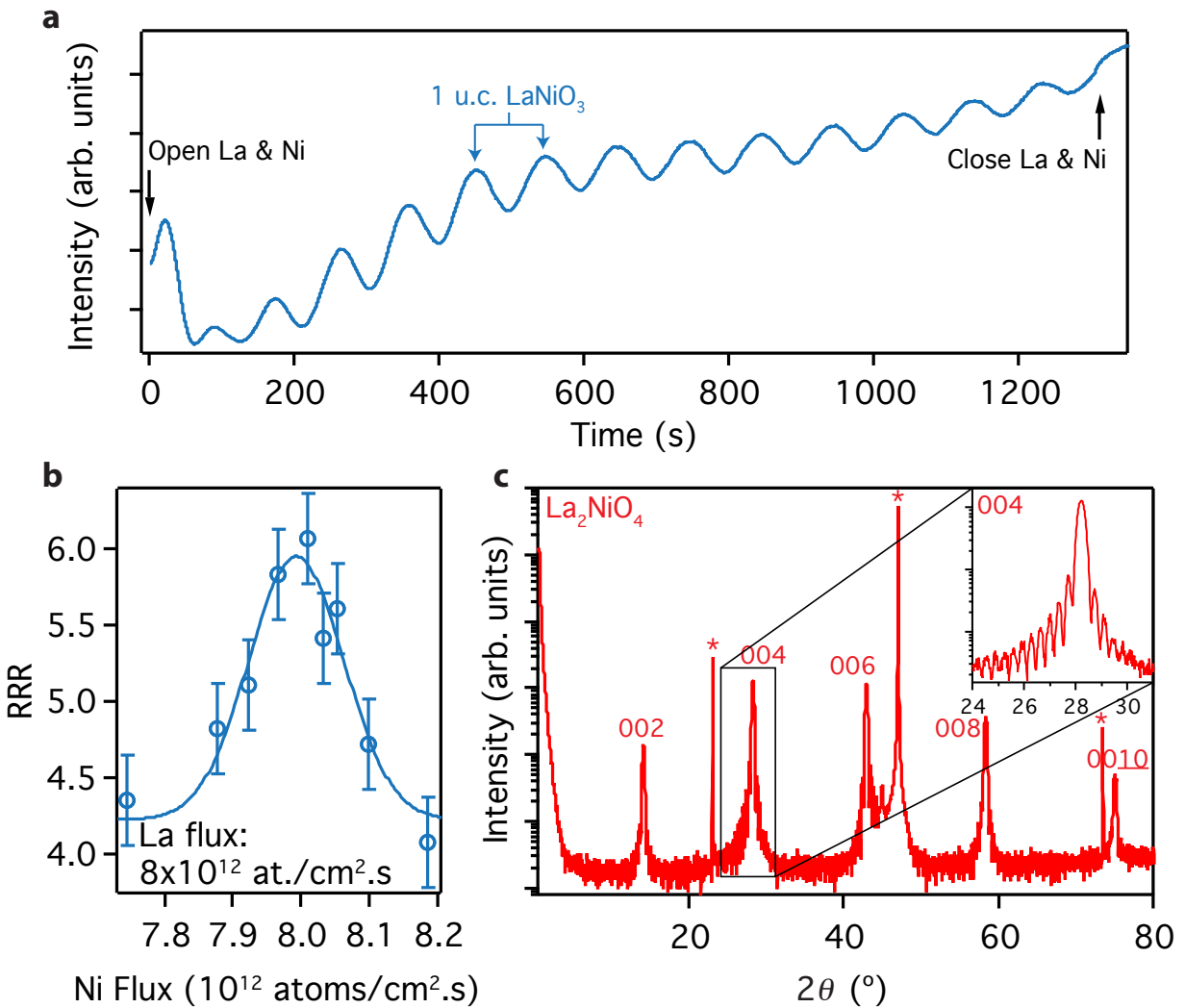

Supplementary Fig. 1. (a) Persistent RHEED oscillations during growth of $\mathrm{LaNiO}_{3}$ by co-deposition of La and Ni, indicating a layer-by-layer growth mode. (b) Dependence of the residual resistivity ratio (RRR) of such films on the estimated flux of $\mathrm{Ni}$ (the flux of La was fixed at $8 \times 10^{12}$ atoms $/ \mathrm{cm}^{2} \mathrm{~s}$ ). (c) X-ray diffraction of a $\mathrm{La}_{2} \mathrm{NiO}_{4}$ film grown by shuttered deposition of $\left[\mathrm{LaO}-\mathrm{NiO}_{2}-\mathrm{LaO}\right]_{40}$ on an LSAT $\left[\left(\mathrm{LaAlO}_{3}\right)_{0.3}\left(\mathrm{Sr}_{2} \mathrm{AlTaO}_{6}\right)_{0.7}\right]$ substrate. The shutter times were taken from the monolayer deposition times determined from our $\mathrm{LaNiO}_{3}$ calibration (and corrected for the different atomic density of the substates), demonstrating the accuracy of our calibration methodology.

a

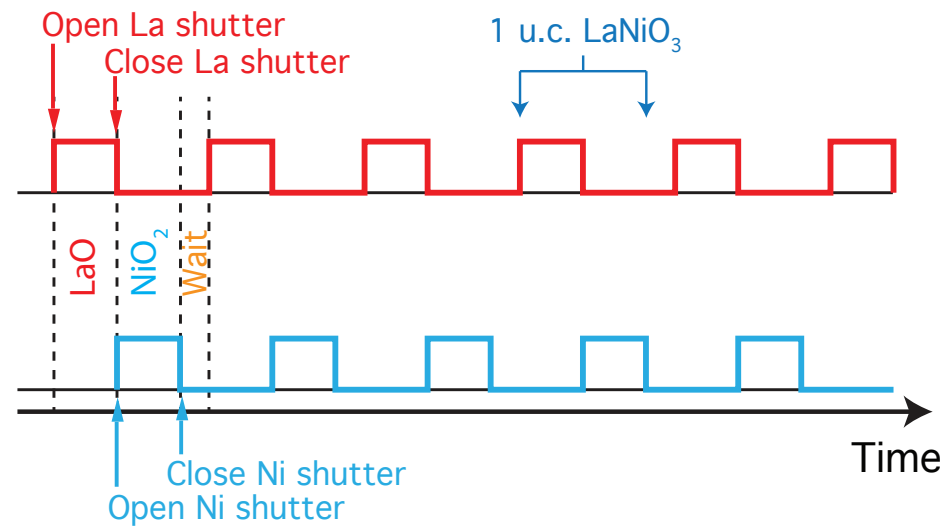

b
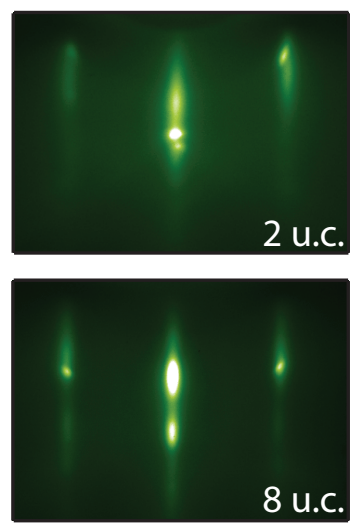

Supplementary Fig. 2. (a) Growth sequence for the $\mathrm{LaNiO}_{3}$ films shown in the main text. Optimal films were achieved in a shuttered growth mode, comprising alternating monolayer deposition of $\mathrm{LaO}$ and $\mathrm{NiO}_{2}$, with a $20 \mathrm{~s}$ wait time following the $\mathrm{NiO}_{2}$ deposition. All samples were terminated with $\mathrm{LaO}$, to maintain a local octahedral crystal field as similar to the bulk as possible, even for the monolayer $\mathrm{LaNiO}_{3}$ sample. (b) Example RHEED patterns (measured along the [110] pseudocubic direction) at the end of such sequences for growth of 2 u.c. (top) and 8 u.c. (bottom) samples. 

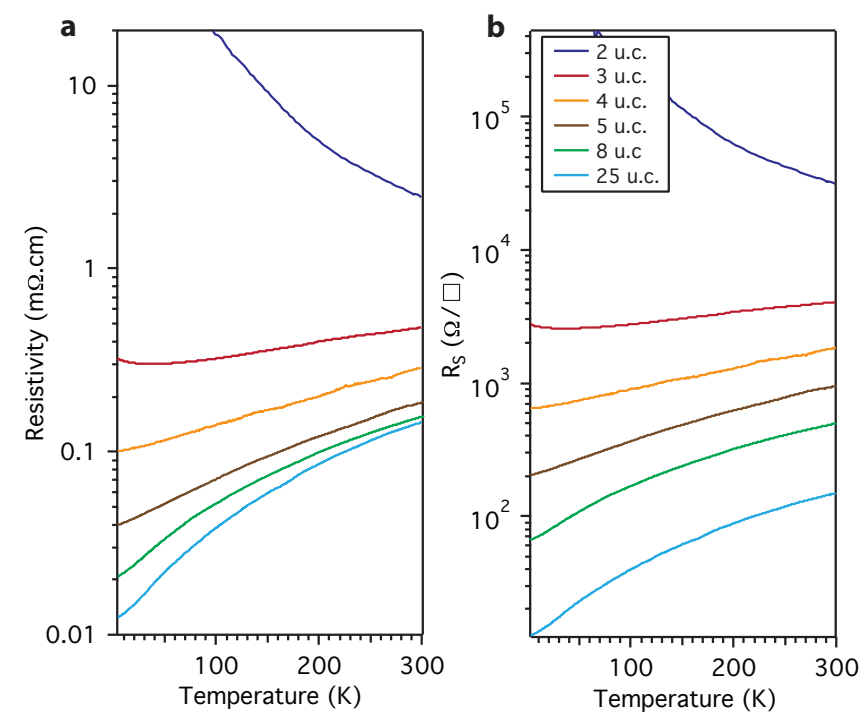

Supplementary Fig. 3. Thickness dependence of the (a) resistivity and (b) corresponding sheet resistance of our $\mathrm{LaNiO}_{3}$ ultrathin films.

as our surface-sensitive ARPES measurements would not then show such a striking change on decreasing from 3 to 2 u.c. film thickness. Rather, we conclude that these oxygen vacancies predominantly develop with time after removing the samples from vacuum following our spectroscopic measurements, consistent with timedependent changes we observe in the resistivity also following the oxygen-annealing experiments. Resistivity measurements performed immediately $(<1 \mathrm{hr})$ after completing ARPES measurements on the 2 u.c. film shown in Fig. 2 of the main text are already insulating. This is supported by the observation of electrostatic charging below $50 \mathrm{~K}$ during the ARPES measurements, indicating an intrinsic insulating behaviour of the 2 u.c. samples.

Moreover, we find that the predominant effect of oxygen vacancies on the ARPES spectra is to increase the non-dispersive background, while the dispersive component remains qualitatively unchanged. By growing a sample under oxygen-deficient conditions $\left(4 \times 10^{-7}\right.$ Torr growth pressure, over an order of magnitude lower ozone partial pressure during growth), we have deliberately driven an extrinsic metal-insulator transition in a 7 u.c. thick sample (Fig. 5(a)). Following background subtraction however (see below), our ARPES measurements reveal a dispersive component remarkably similar to all of our other samples that are thicker than 3 u.c. (Fig. 5(b,c)). Crucially, this has a strikingly different form to the insulating state of the 2 u.c. sample (Fig. 2 of the main text), indicating that the spectral features of the 2 u.c. sample are not simply signatures of oxygen deficiency. Thus, unlike transport, ARPES allows us to discriminate between an intrinsic and extrinsic MIT. The consistent critical thickness that we find for the thickness- driven MIT from transport and ARPES in our main study confirms that we are probing an intrinsic transition by both transport and spectroscopy here.

To additionally rule out islanding as the origin of the transport MIT in ultrathin samples, we performed atomic force microscopy (AFM) using a Veeco Dimension 3100 AFM operated in tapping mode (Fig. 2 of the main text and Fig. 6). Even for a single u.c. thick $\mathrm{LaNiO}_{3}$ film, we find high-quality step-and-terrace surfaces with pseudocubic unit cell height steps across an extended field of view (Fig. 6). The terraces are smooth, with a characteristic r.m.s. roughness better than $15 \%$ of a pseudocubic unit cell. This confirms that the metal-insulator transition we observe at a film thickness of 2 u.c. is intrinsic, and does not occur due to a loss of percolation driven by island formation. The high surface quality of our films is further confirmed by sharp RHEED patterns (Fig. 2(b)).

To confirm this, and to check that the surface quality did not degrade during sample transfer or with time during our spectroscopic measurements, we performed lowenergy electron diffraction (LEED) after completing our photoemission experiments. As shown in Fig. 7, the samples showed sharp integer-order diffraction spots, characteristic of a clean and well ordered surface. We additionally observe $\sqrt{2} \times \sqrt{2}$ diffraction spots (indicated by a red arrow in Fig. 7). These are relatively weak, sometimes barely observable, for all incident electron energies (Fig. 8), and we attribute these to weak rhombohedral distortions that define the bulk structure of $\mathrm{LaNiO}_{3}$. We additionally observe weak signatures of a corresponding band folding about the $(\pi, 0)-(0, \pi)$ line in our measured ARPES Fermi surface maps. We find no evidence, however, for an increase in strength of these features in the thinner samples either in our electron diffraction or ARPES maps, as might have been expected if structural distortions in the thinest films played a significant role in the metal-insulator transitions observed here.

For all samples, transport measurements were performed after the spectroscopic study was completed, using a home-built linear four-point-probe setup, with the probes aligned parallel to the twin boundaries present in the $\mathrm{LaAlO}_{3}$ substrates. We found no evidence for systematic variation in resistivity when measuring parallel or perpendicular to the twin boundaries, and so conclude that they do not significantly influence our resistivity measurements or limit the metallicity of the films.

\section{SUPPLEMENTARY DISCUSSION 2. ANGLE- RESOLVED PHOTOEMISSION SPECTROSCOPY ANALYSIS}

Our ARPES measurements from the thicker films match our simulated bulk Fermi surface from densityfunctional theory calculations of $\mathrm{LaNiO}_{3}$ well, as discussed in the main text. To quantify this, we show in Fig. 9 extracted Fermi momenta from our measured Fermi surfaces as well as the calculated Fermi surface for 


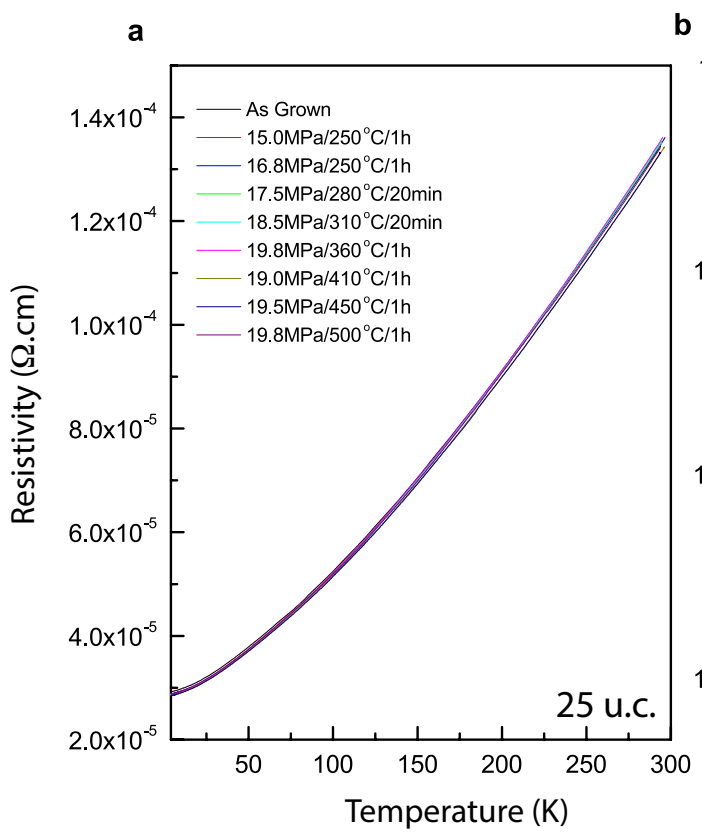

b

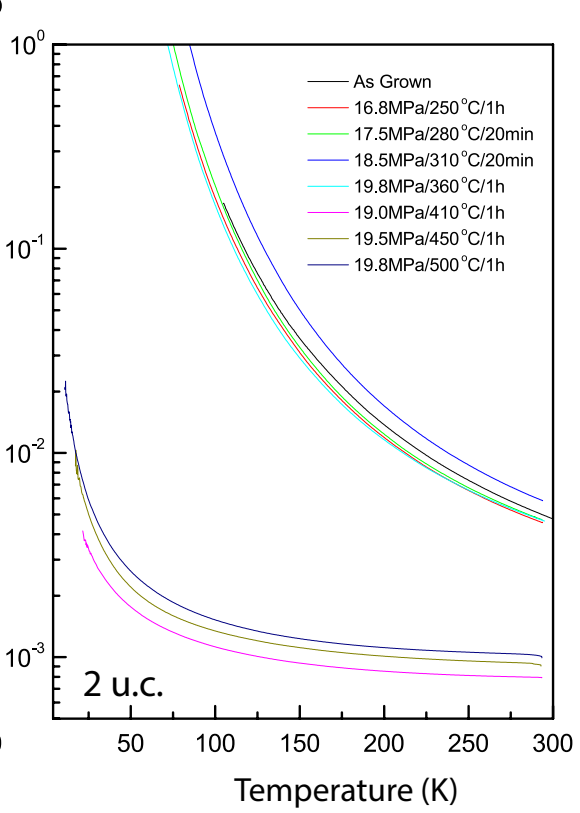

Supplementary Fig. 4. Effect on transport of sequential high-pressure oxygen anneals of (a) 25 u.c. and (b) 2 u.c. $\mathrm{LaNiO}_{3}$ under the conditions listed in the captions.
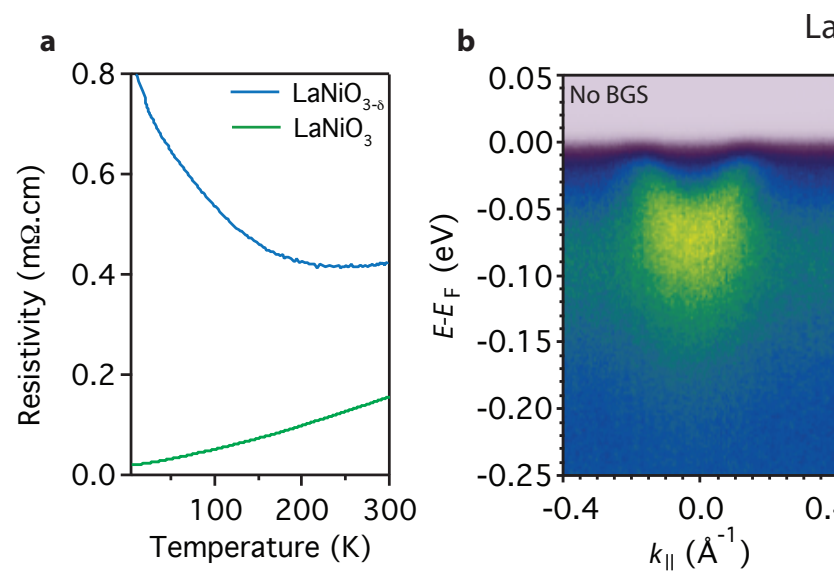

$\mathrm{LaNiO}_{3-\delta}$

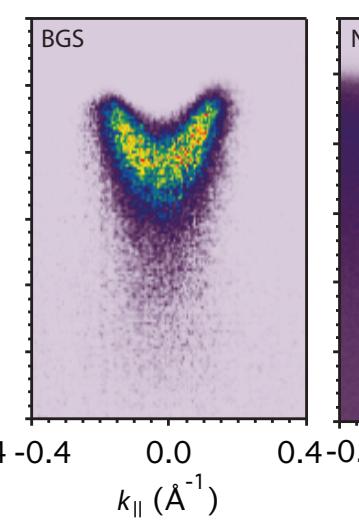

c
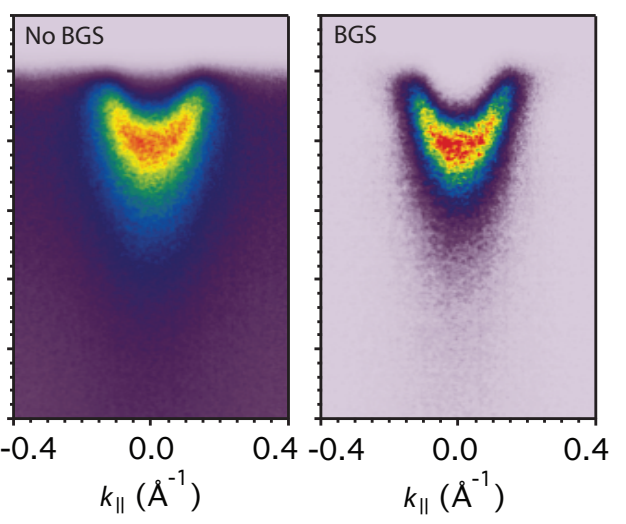

Supplementary Fig. 5. (a) Transport from an oxygen deficient 7 u.c. and fully oxygenated 8 u.c. sample, showing how oxygen vacancies can drive an extrinsic metal-insulator transition. Our spectroscopic measurements (b,c) show an increased background in the oxygen deficient sample. Following our background subtraction procedure, however, the spectra look very similar, revealing almost identical intrinsic spectral functions in both cases.

the central $k_{z}$ value probed in our experimental configuration. These agree very well for the sections of the hole pockets which do not disperse significantly in $k_{z}$. We find small deviations from the calculated Fermi surface for bands which have substantial $k_{z}$ dispersion (e.g., the calculated electron pocket at the zone centre is slightly smaller than the extracted $k_{F}$ in Fig. $9(\mathrm{~b})$, and the spectral weight in our measurements here is also rather diffuse instead of giving a sharp Fermi surface). Such features are entirely consistent with the surface sensitivity of photoemission, causing us to sample a finite width in $k_{z}$, as also captured in our simulated Fermi surface spectra which include this effect, shown on a false colour scale beneath the extracted points in Fig. 9 as well as in Fig. 1 of the main text.

In all samples, we observed a momentum-independent background contributing spectral weight up to the Fermi level (see, e.g., Fig. 10(a)). Such backgrounds are commonly observed in photoemission spectra and here likely result from the broad tail of intensity of the $\mathrm{Ni} \mathrm{t}_{2 g}$ states, as well as some defect states that appear to contribute a non-dispersive impurity band peaked approxi- 
a

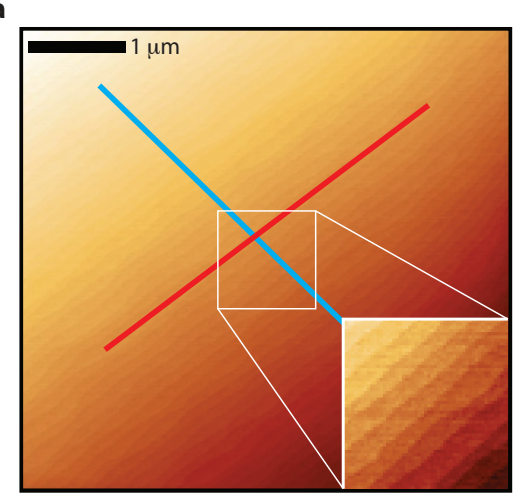

b

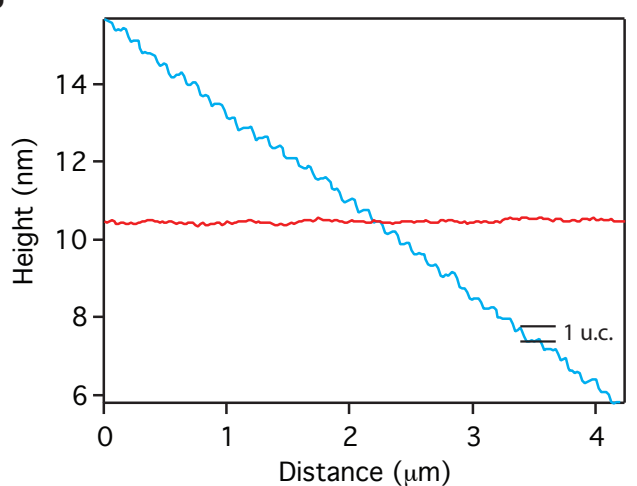

Supplementary Fig. 6. (a) Atomic force microscopy of a single u.c. thick sample, measured across a $5 \times 5 \mu \mathrm{m}$ field of view. A magnified $1 \times 1 \mu \mathrm{m}$ region is shown inset. (b) Line cuts along the coloured paths shown in (a), revealing a step-and-terrace surface with pseudocubic unit cell height steps and smooth terraces.
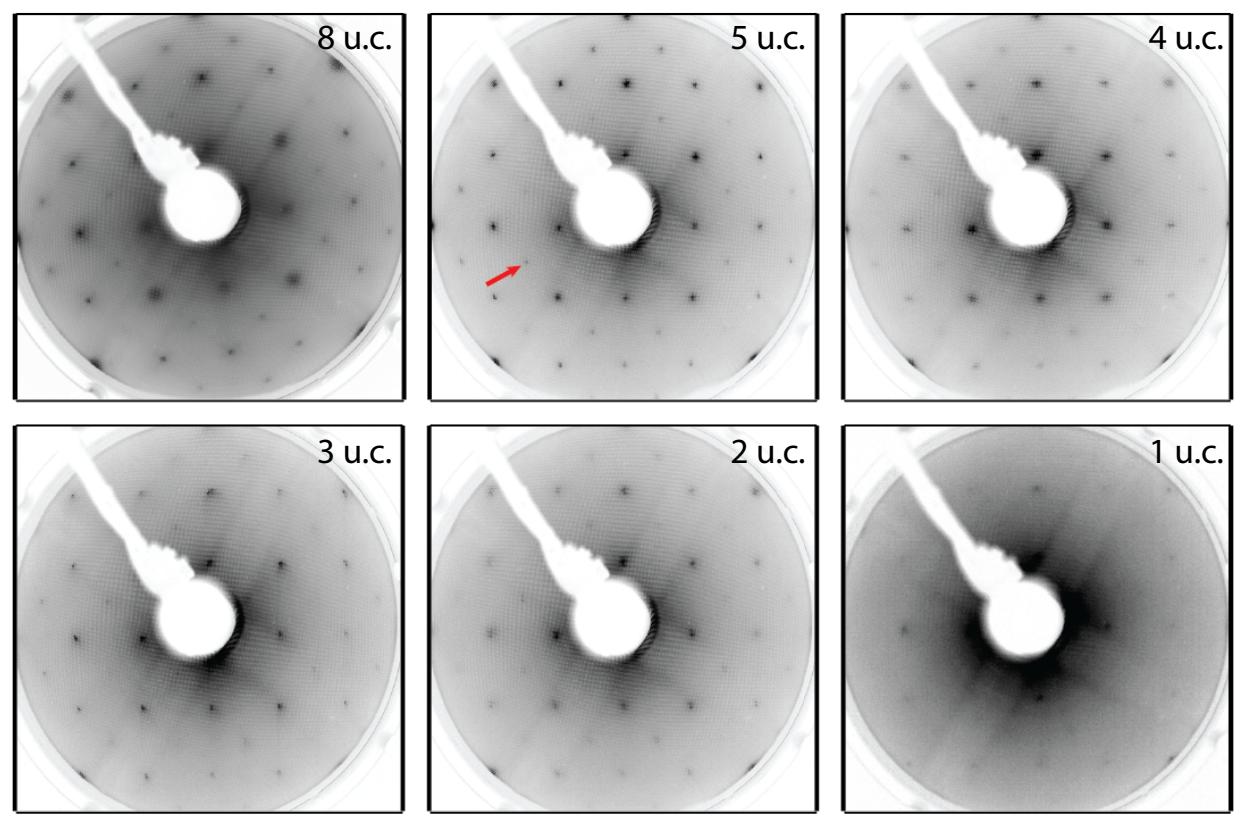

Supplementary Fig. 7. Low-energy electron diffraction, recorded with a beam energy of $150 \mathrm{eV}$, from 8, 5, 4, 3, 2, and 1 u.c. thick samples. The measurement temperature was $20 \mathrm{~K}(3,5$, and 8 u.c.), $50 \mathrm{~K}$ (2 and 4 u.c.), and $330 \mathrm{~K}$ for the single u.c. thick sample to prevent charging. A representative $\sqrt{2} \times \sqrt{2}$ diffraction spot is indicated by the red arrow for the 5 u.c. thick sample.
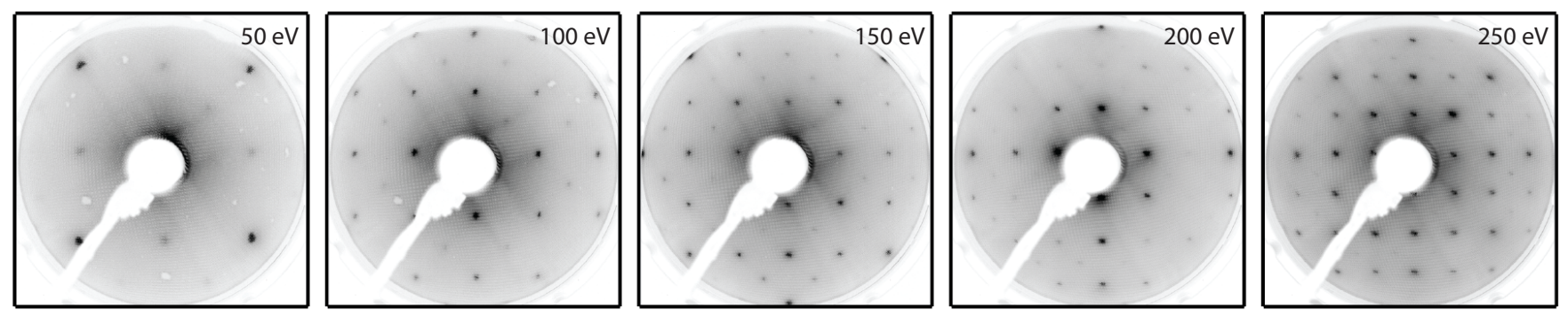

Supplementary Fig. 8. Incident electron energy dependence of low-energy electron diffraction from a 5 u.c. sample, measured at $20 \mathrm{~K}$. 


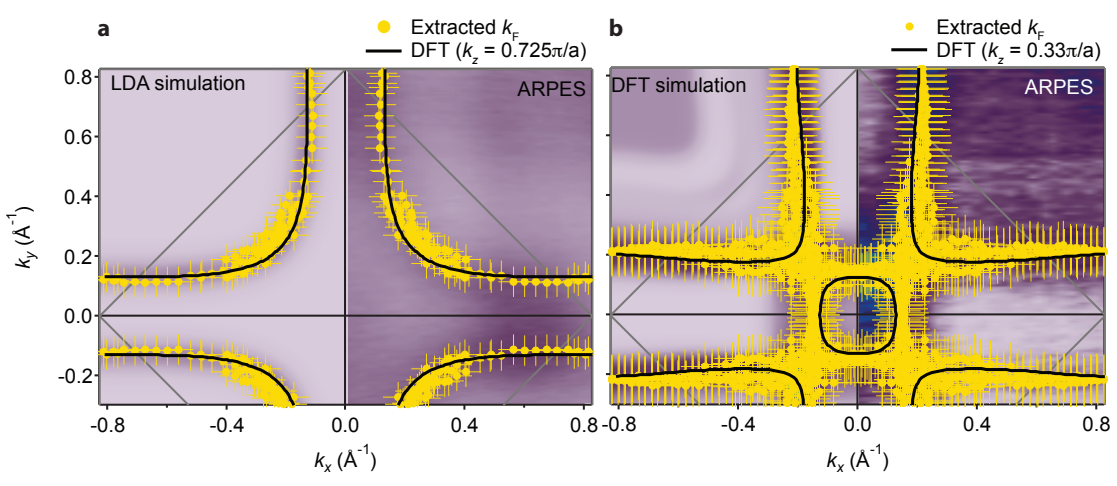

Supplementary Fig. 9. Simulated (left) and measured (right) Fermi surface for (a) He I $\alpha\left(k_{z} \approx 0.7 \pi / a\right)$ and (b) He II $\alpha$ $\left(k_{z} \approx 0.3 \pi / a\right)$ excitation, reproduced from Fig. 1 of the main text. Overlaid are Fermi momenta extracted from our experimental measurements and four-fold symmetrized (yellow points) and the density-functional Fermi surface corresponding to the central $k_{z}$ value probed (black line).
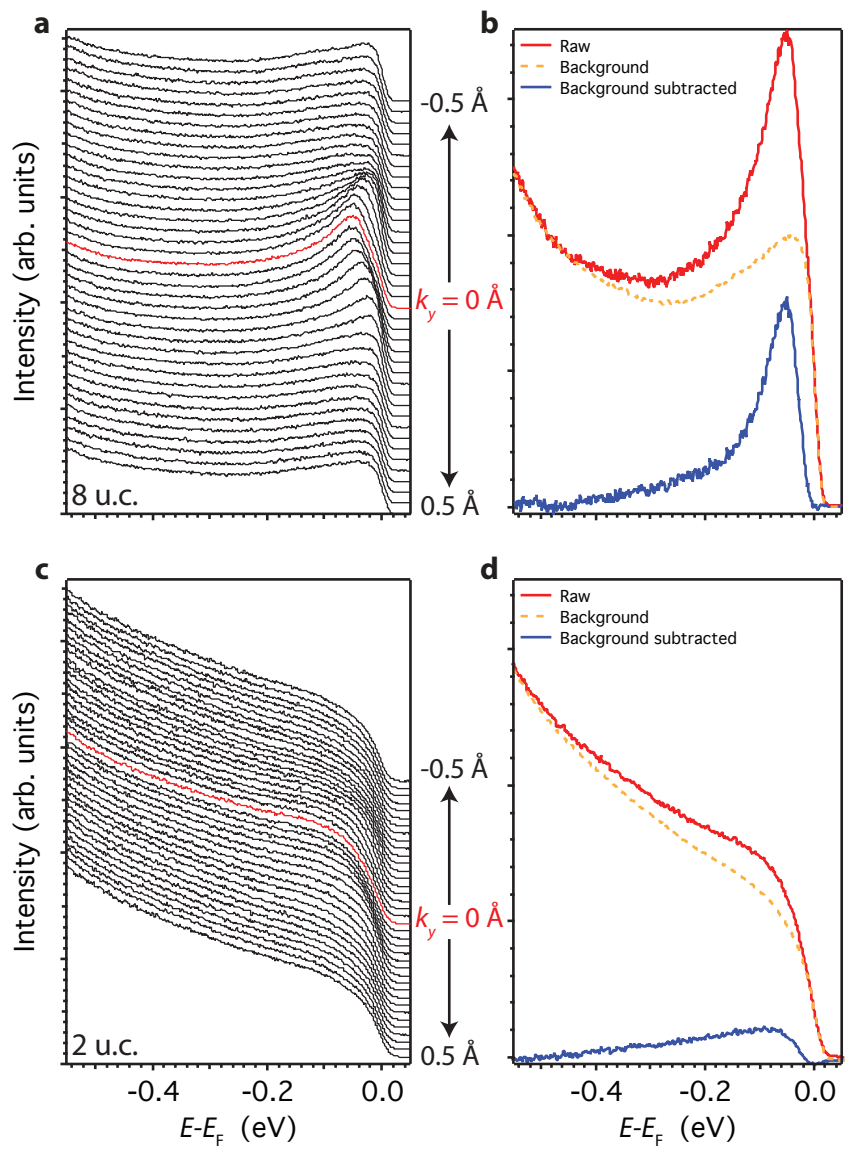

Supplementary Fig. 10. Raw EDCs along $\left(0.5 \pi, k_{y}, 0.7 \pi\right)$ from an (a) 8 u.c. and (c) 2 u.c. thick sample. (b) 8 u.c. and (d) 2 u.c. momentum-independent background (orange dashed line), calculated by integrating the raw EDCs between $0.4<k_{y}<0.6 \AA^{-1}$, and showing good agreement with the $k_{y}=0$ EDC (red line) at high binding energies. The corresponding background-subtracted EDC is also shown (blue). mately $0.1 \mathrm{eV}$ below the Fermi level, and in the thinnest films could additionally reflect a decrease in total spectral weight coming from the film as the probing depth of our measurements starts to exceed the total $\mathrm{LaNiO}_{3}$ thickness. To remove this background, which contributes somewhat larger spectral weight relative to the dispersive $e_{g}$ states in the thinner samples (Fig. 11), we applied a momentum-independent background subtraction to the data shown in Figs. 1(d), 2(b-f), and 3(a) in the main manuscript. This background was determined by integrating EDCs between $0.4<k_{y}<0.6 \AA^{-1}$. Subtraction of this background (Fig. 10(b)) permits extracting the intrinsic spectral function of relevance to our study. The result of applying this procedure to all of the spectra shown in the main text is summarized in Fig. 11. We stress that all dispersions are also apparent in the raw data, even for the 2 u.c. film, and the background subtraction merely makes these features easier to visiualise and allows for a consistent definition of the effective quasiparticle residue.

To quantitatively describe the loss of quasiparticle coherence, we fit two Lorentzian peaks to the EDCs extracted at the Fermi wavevector. Physically, these account for the coherent and incoherent components of the spectral function, the former fixed to zero energy as shown in Fig. 3(b) of the main text. From the areas, $A_{i}$, of these, we define an effective quasiparticle residue as $Z^{\prime}=A_{\text {coh }} /\left(A_{c o h}+A_{\text {inc }}\right)$. Note, this is not the true residue, $Z$, but rather reflects the reduction of the quasiparticle weight relative to that bulk, the quantity of interest as we reduce the film thickness down to nanoscale dimensions. The loss in quasiparticle coherence at 2 u.c. is accompanied by an increase in the apparent bandwidth, as shown in Fig. 3 of the main text and also apparent from the larger slope of MDC fits near the Fermi level in the 2 u.c. sample (Fig. 12). The resilience of the MDC linewidth at the Fermi level (Fig. 13) rules out localization as the origin of the corresponding thickness-driven metal-insulator transition. 
a

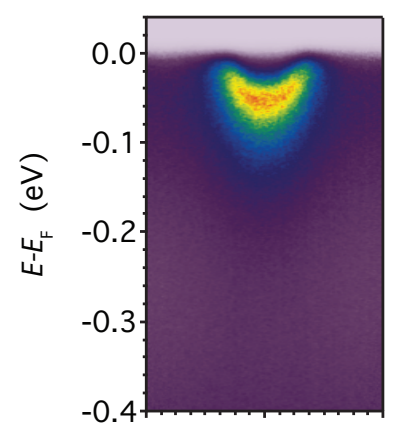

b

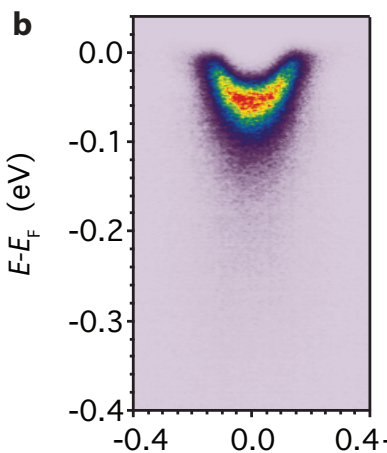

5 u.c.
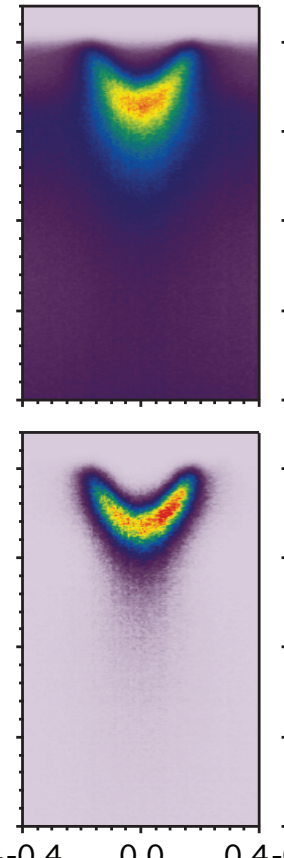

4 u.c.
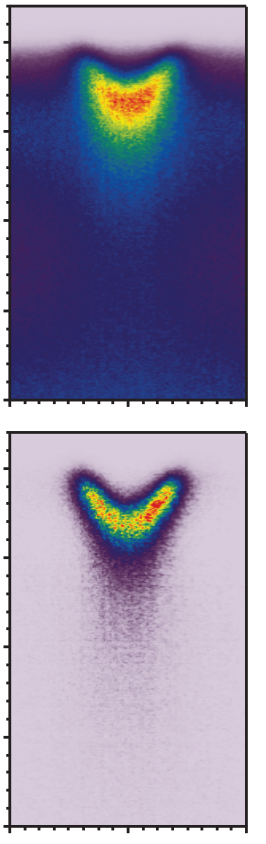

3 u.c.
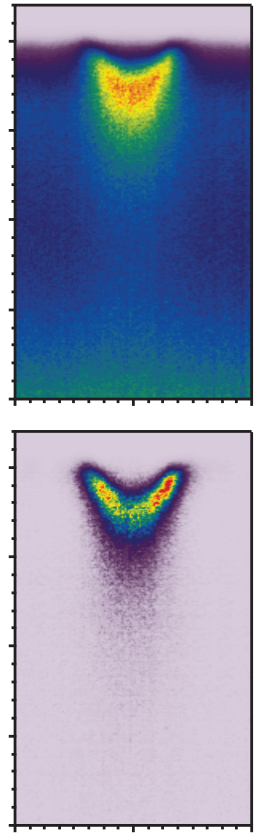

2 u.c.
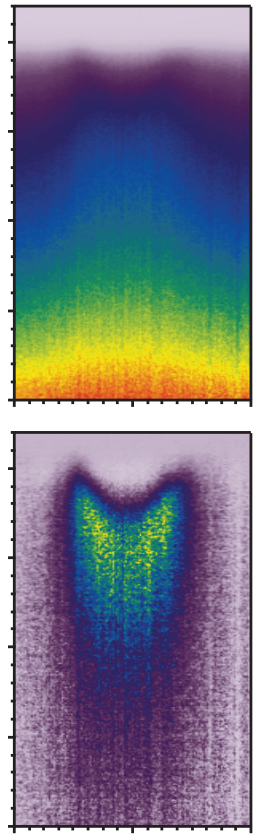

1 u.c.

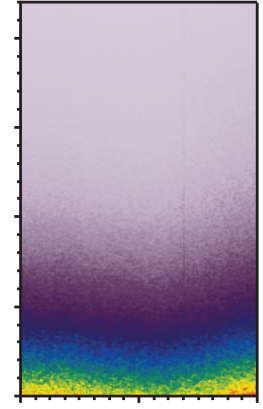

$\frac{\sigma}{\sigma}$
$\frac{\pi}{0}$
$\frac{1}{0}$

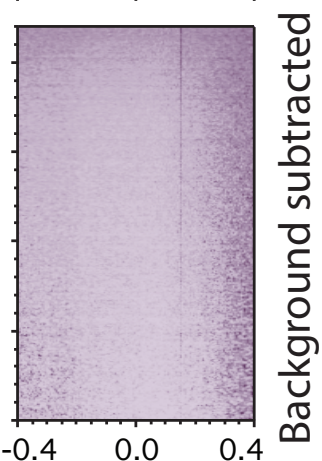

Supplementary Fig. 11. (a) Raw ARPES spectra along $\left(0.5 \pi, k_{y}, 0.7 \pi\right)$ from 8, 5, 4, 3, 2, and 1 u.c. thick samples. (b) Equivalent spectra after subtraction of a momentum-independent background, determined by integrating EDCs between $0.4<$ $k_{y}<0.6 \AA^{-1}$.

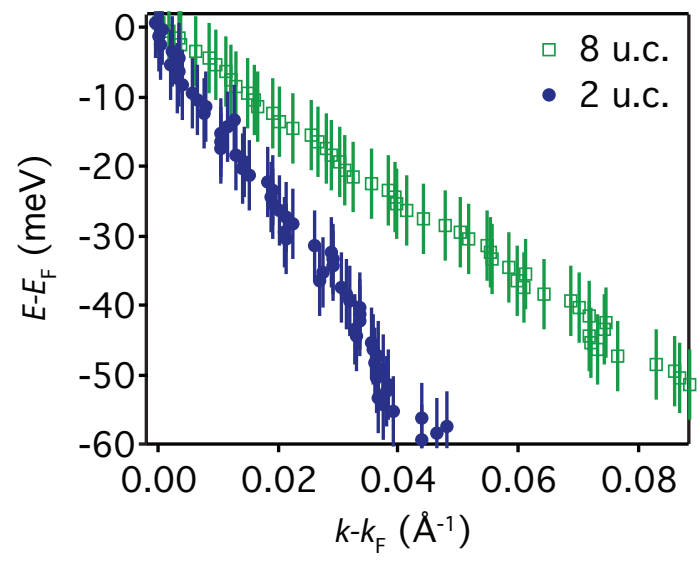

Supplementary Fig. 12. Dispersion extracted from MDCs of the 8 u.c. and 2 u.c. samples shown in Fig. 2 of the main text.

\section{SUPPLEMENTARY DISCUSSION 3. DENSITY- FUNCTIONAL THEORY CALCULATIONS.}

The ab-initio calculations shown in Fig. 1 of the main text were calculated within density-functional theory using the Wien2k code, ${ }^{5}$ and the Perdew-Burke-
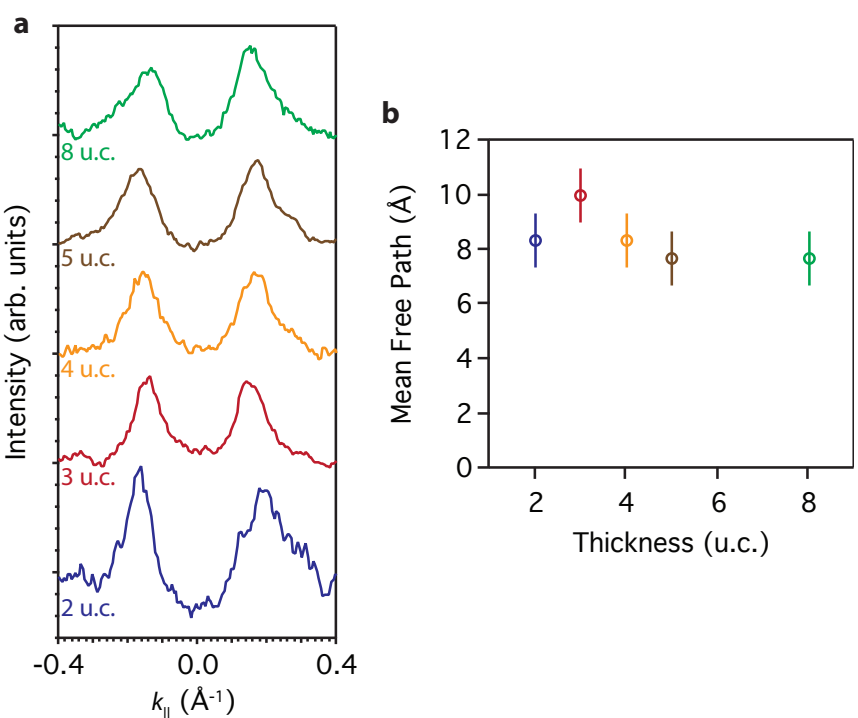

Supplementary Fig. 13. (a) MDCs at the Fermi level, and (b) corresponding inelastic mean free path derived from the average linewidth of Lorentzian fits to these MDCs.

Ernzerhof exchange-correlation functional. ${ }^{6}$ An idealized cubic structure was assumed, with a relaxed lattice con- 
stant of $a=3.84 \AA$. Previous calculations have shown that the rhombohedral distortions of $\mathrm{LaNiO}_{3}$ lead to a zone folding, ${ }^{7}$ and we find evidence for this in our measured Fermi surface maps (Fig. 1(a) of the main text). Spectral signatures of this are, however, weak, as are the corresponding half-order spots observed in our LEED experiments (Fig. 7) and so we restrict our calculations to a cubic symmetry here for simplicity.

To determine the values of $k_{z}$ to compare the calculations to our experimental data, we employ a free-electron final state model:

$$
k_{z}=\sqrt{2 m_{e} / \hbar^{2}}\left(V_{0}+E_{k} \cos ^{2} \theta\right)^{1 / 2}
$$

where $m_{e}$ is the free electron mass, $E_{k}$ the kinetic energy of the outgoing photoelectron, $\theta$ the emission angle and $V_{0}$ the inner potential. This latter quantity was taken as $10 \mathrm{eV}$, as determined from the photon energydependent ARPES measurements of Eguchi et al.. ${ }^{8}$ At normal emission $\left(\theta=k_{\|}=0\right)$, this gives $k_{z}=0.73 \pi / a$ for $\mathrm{He} \mathrm{I} \alpha$ and $k_{z}=0.33 \pi / a$ for He II $\alpha$ light. For simulation of the measured Fermi surface maps (Fig. 1a of the main manuscript) we additionally applied a $k_{z^{-}}$ integration to account for final-state broadening arising from the surface sensitivity of ARPES. We described the $k_{z}$ broadening by a Lorentzian, centred at $k_{z}=0.73 \pi / a$ or $k_{z}=0.33 \pi / a$, and with a full width at half maximum $\delta k_{z}=1 / \lambda$ where $\lambda$ is the inelastic mean free path of the photoelectrons, which we take here as $\sim 4 \AA$.
1 Blank, D. H. A. Private communication.

2 Sánchez, R. D. et al. Metal-insulator transition in oxygendeficient $\mathrm{LaNiO}_{3-x}$ perovskites. Phys. Rev. B 54, 1657416578 (1996).

3 Zhou, J.-S., Goodenough, J. B., Dabrowski, B., Klamut, P. W. \& Bukowski, Z. Enhanced Susceptibility in $\mathrm{LNiO}_{3}$ Perovskites $\left.\left(L=\mathrm{La}, \mathrm{Pr}, \mathrm{Nd}, \mathrm{Nd}_{0.5} \mathrm{Sm}\right) 0.5\right)$. Phys. Rev. Lett. 84, 526-529 (2000).

${ }^{4}$ Horiba, K. et al. Electronic structure of $\mathrm{LaNiO}_{3}$ : An in situ soft x-ray photoemission and absorption study. Phys. Rev. $B$ 76, 155104 (2007).
5 Blaha, P., Schwarz, K., Madsen, G., Kvasnicka, D. \& Luitz, J. Wien2k package. available at http:// www.wien2k.at.

${ }^{6}$ Perdew, J. P., Burke, K. \& Ernzerhof, M. Generalized gradient approximation made simple. Phys. Rev. Lett. 77, 38653868 (1996).

7 Hamada, N. Electronic band structure of $\mathrm{LaNiO}_{3}$. J. Phys. Chem. Solids. 54, 1157-1160 (1993).

${ }^{8}$ Eguchi, R. et al. Fermi surfaces, electron-hole asymmetry, and correlation kink in a three-dimensional Fermi liquid $\mathrm{LaNiO}_{3}$. Phys. Rev. B 79, 115122 (2009). 